\title{
Etudes Industrielles de vibrations sous écoulement dans des réseaux de tuyauteries
}

\author{
Industrial vibration studies on flow in pipe networks
}

par D. Seligmann, I. Fournier

Electricité de France

Direction des Etudes et Recherches, Clamart

To improve the allowance made in the design stage for vibratory phenomena affecting main lines and nozzles under operating conditions have led Electricite de France to develop special engineering codes and study methodologies. The basic aim of the various actions undertaken has been to locate the causes of fractures of cracking of piping in auxiliary circuits, examine the corrective actions taken, and define industrial vibration prevention practice.

So far, vibration problems have been detected during start-up tests. As regards nozzles, the cracks sometimes appear rapidly, and are in this case treated during the installation testing period, as called for by the RCC-M. Further more, the problem posed by vibration fatigue is hard to solve, because the operating configuration that gave rise to the problem must be known.

Evolution of EDF's acceptance guide involves incorporation on the vibration acceptance criterion for piping systems. Knowing the operation of the circuit, we predict the dynamic loads generated by the flow, to predict the vibratory behavior of the piping, in particular that of nozzles, and so estimate the design margins with respect to the vibration risk either using the current acceptance criteria or a damage analysis. Two methodologies based on the modeling and calculation options of the CIRCUS code are proposed. One concerns studies based on operating design data and the other studies based on the operating diagnostic. The latter is based on an approach combining numerical modeling and on-site tests.

\section{I $\square$ INTRODUCTION}

- 1.1 La problématique des études vibratoires de tuyauteries

Dès le début des années 1980, Electricité de France mettait l'accent à la fois sur la nécessité d'améliorer la prise en compte à la conception des phénomènes vibratoires sur les lignes principales et les piquages et sur l'intérêt de vérifier le bon comportement vibratoire des points singuliers, zones potentielles de vibration, dans des conditions de fonctionnement pénalisantes vis-à-vis de ce phénomène. Différentes actions ont été engagées ayant pour objet la recherche des causes de ruptures ou de fissurations de tuyauteries sur les circuits de sûreté avec l'examen des remèdes apportés et la détermination de solutions industrielles pour réduire les vibrations.

De ces études, il était ressorti que lorsqu'un problème vibratoire était détecté, il était résolu localement en agissant la plupart du temps sur la réponse mécanique du système et que, dans certains cas particuliers parfaitement identifiés, il était possible d'intervenir directement sur la source origine des vibrations.
Le bilan des études passées montrait alors qu'aucun critère de conception ne pouvait être directement utilisable industriellement pour prendre en compte les vibrations lors des études de conception.

1.2 Les enjeux : exemple des piquages des circuits importants pour la sûreté.

Les circuits importants pour la sûreté (IPS) sont conçus et exploités pour assurer une fonction donnée. A cette fin, ils doivent rester intègres. Tout matériel peut devenir défaillant, et les conséquences vis-à-vis de la sûreté s'apprécient par rapport aux exigences du Rapport de Sûreté et/ou des Règles Générales d'Exploitation. Ces exigences tiennent compte à la fois des conditions de fonctionnement et de la fonction assurée par le circuit.

Du point de vue disponibilité, et en fonctionnement normal, les Spécifications Techniques d'Exploitation permettent de faire face à la défaillance d'un piquage. Mais les délais de réparation ou de repli peuvent être supérieurs aux délais autorisés et de ce fait imposer une indisponibilité de la tranche pouvant aller jusqu'à plusieurs jours. 


\section{II — MÉTHODOLOGIES POUR LES ÉTUDES DE VIBRATIONS DE TUYAUTERIES.}

\section{- 2.1 Un guide-type de réception}

La tenue mécanique des tuyauteries des circuits de sûreté n'est actuellement pas vérifiée au stade de la conception visà-vis des sollicitations vibratoires en fonctionnement, notamment vis-à-vis des sollicitations engendrées par les fluctuations de pression et de débit dues aux organes de réglage. Une méthodologie applicable aux mesures de vibrations des tuyauteries a été élaborée. Elle s'appuie sur l'expérience acquise lors des paliers précédents et concerne l'aspect détection du problème. Les vérifications sont réalisées lors des contrôles de fin de montage et lors des essais périodiques. Rassemblées au sein d'un guide-type, ses dernières évolutions s'appuient sur la norme américaine ANSIOM3 [1] pour les collecteurs et sur les études menées par EDF $[2,3]$ sur les piquages.

\subsection{Norme de I'ASME ANSI-OM3 de réception vibratoire}

La norme ASME ANSI-OM3 définit un critère de réception vibratoire des lignes de tuyauteries. Elle établit une relation entre la vitesse vibratoire maximum d'un tronçon de tuyauterie et la contrainte alternée admissible pour le matériau. Cette relation fait intervenir plusieurs coefficients dont les valeurs dépendent du tracé de l'isométrique de la ligne, des conditions de supportage, de la présence de masses additionnelles et de l'effet de concentration de contrainte dans les zones singulières.

La norme précise la fourchette dans laquelle sont compris ces coefficients, ainsi que la limite d'endurance du matériau qui conduit à une valeur seuil enveloppe de vitesse vibratoire admissible fixée à $12 \mathrm{~mm} / \mathrm{s}$. Cette valeur seuil de $12 \mathrm{~mm} / \mathrm{s}$, appliquée en terme de valeur efficace est adaptée à l'ensemble des circuits équipant les centrales nucléaires françaises. L'expérience montre qu'effectivement les fissurations par fatigue vibratoire constatées ont exigé des niveaux de vibrations nettement plus élevés. C'est cette valeur qu'on applique lors de la réception des circuits importants pour la Sûreté. En cas de dépassement de ce critère, on revient à la formule de base proposée par la norme et on adapte les coefficients en fonction des caractéristiques propres du tronçon, ce qui permet de relâcher le critère et de juger acceptables dans certains cas les vibrations de la ligne sans autres investigations (essais, calculs, modifications).

La formule actuellement utilisée par EDF est dérivée de celle proposée par la norme américaine, et cela afin d'expliciter le caractère aléatoire des vibrations par l'introduction d'un facteur de pic. Elle aboutit au même critère moyennant certaines interprétations ainsi que quelques corrections relatives aux valeurs enveloppes des coefficients.

Par ailleurs, une étude visant à expliciter les marges propres à chacun des coefficients a été entreprise. Afin de s'assurer de la validité des résultats obtenus sur l'ensemble des isométries testées, on a vérifié la concordance des résultats analytiques et des résultats numériques obtenus par le code CIRCUS dans le cas d'excitations aléatoires imposées sur des oscillateurs simples et sur un oscillateur couplé fluide-structure. Ces excitations sont du type effort, déplacement, pression ou débit selon la nature de l'oscillateur considéré.

\section{- 2.3 Méthodologies de calcul pour les études}

A l'issue des essais de réception, et si les critères sont dépassés, on effectue un calcul de réponse de la ligne sous l'excitation aléatoire due aux sources hydroacoustiques présentes sur le circuit. On vérifie alors l'acceptabilité ou la non acceptabilité des vibrations des lignes en fonctionnement. Pour mener à bien ces études, deux approches d'étude ont été développées. C'est pour répondre à ces démarches que le code CIRCUS a été développé.

\subsubsection{Démarche à partir des données de conception.}

Les connaissances actuelles [4] permettent d'estimer les marges de dimensionnement vis-à-vis du risque vibratoire à basses fréquences des réseaux de tuyauteries avec les critères actuels de réception ou avec des critères dérivés. Cette évaluation s'appuie sur les compétences acquises lors de la détermination des sources d'excitation acoustique. Ces sources sont élaborées à partir de la connaissance du fonctionnement de l'installation et de la géométrie des composants (vanne, pompe, diaphragme,... ).

La modélisation acoustique et vibratoire des lignes de tuyauterie et le calcul de dommage basé sur l'analyse des contraintes permettent alors de vérifier les critères de vitesses vibratoires et d'endommagement (figure 1).

\section{Analyse du fonctionnement. \\ Calcul de l'écoulement permanent. \\ Calcul des sources acoustiques des composants (pompe, vanne,... ). \\ Calcul de la réponse acoustique et mécanique du réseau de tuyauteries. \\ Vérification des critères de vitesse vibratoire. \\ Calcul des contraintes mécaniques. Vérification de la durée de vie.}

\section{Méthodologie à partir des données de conception du} fonctionnement.

\subsubsection{Démarche à partir du diagnostic en exploitation.}

En exploitation, l'évaluation est basée sur une approche intégrée de la modélisation numérique et des essais sur site (figure 2). Il est possible d'effectuer un calcul inverse utilisant des mesures en entrée afin d'identifier certaines caractéristiques mal connues, qui pourront elles-mêmes être archivées et réutilisées pour d'autres études.

Identification expérimentale des sources acoustiques sur site.

Identification des régimes de fonctionnement pénalisants.

Identification des conditions limites acoustiques et mécaniques.

Calcul de la réponse acoustique et mécanique du réseau de tuyauteries.

Calcul des contraintes mécaniques.

Vérification de la durée de vie.

2. Méthodologie à partir du diagnostic en exploitation. 


\section{III — L'OUTIL CIRCUS POUR LES ÉTUDES VIBRATOIRES DES CIRCUITS DE TUYAUTERIES}

\section{- 3.1 Présentation générale}

Le code CIRCUS traite du comportement hydraulique, acoustique, mécanique statique, sismique et vibratoire des réseaux de tuyauteries soumis à des excitations induites par l'écoulement, c'est-à-dire dans les conditions du fonctionnement. Centré sur cette application, le code CIRCUS permet, en premier lieu, de calculer l'écoulement permanent, puis d'évaluer les sources acoustiques et les chargements dynamiques associés et, en définitive, de déterminer les niveaux de réponse acoustique et vibratoire le long du réseau. Il est dès lors possible de déterminer les phénomènes acoustiques ou vibratoires susceptibles de se propager, de les localiser, de les quantifier et enfin d'évaluer les risques d'endommagement ou de faire évoluer le comportement du circuit en modifiant les paramètres de dimensionnement ou de fonctionnement. Le code CIRCUS permet de vérifier que les modifications proposées lors d'études de diagnostic restent compatibles avec les aspects de conception réglementaire d'usage dans le domaine nucléaire [5].

Conçu en tant que code applicatif pour effectuer des études de vérification de la conception vibratoire, le code CIRCUS utilise une base de données de singularités acoustiques passives (vannes, diaphragmes, changements de section,...) ou actives (pompes,...) pour reconstituer les sources d'excitation. Cette banque de données rassemble d'une part des formulations analytiques adimensionnalisées vis-à-vis de la géométrie et des conditions de fonctionnement et d'autre part des résultats d'essais relatifs à des matériels particuliers du parc nucléaire français (pompe alimentaire, pompe RIS, pompe primaire,... ) obtenus en fonctionnement sur site ou sur boucles d'essais. Ces sources obtenues sur site peuvent être analysées avec le code, avant d'être stockées dans la base de données, ce qui assure le retour d'expérience des connaissances acquises sur les mécanismes de génération et de propagation du bruit et des vibrations dans les circuits. Elles sont alors mises à la disposition de l'utilisateur pour les besoins de ses études, de façon fiable et transparente.

Le module de calcul permet une simulation du comportement vibratoire d'un réseau de tuyauteries soumis à des sollicitations dynamiques réelles via la prise en compte de mesures issues d'essais sur site.

Son domaine d'utilisation complémentaire est celui de l'identification de caractéristiques mal connues telles que des conditions limites (supportages mécaniques par exemple) ou telles que des efforts ou des chargements dynamiques sur une portion de circuit en fonctionnement.

Le réseau de tuyauteries est modélisé par la théorie des poutres et des ondes planes. La méthode de calcul est basée sur une décomposition élémentaire mixte de matrices de transfert - éléments finis connue sous le nom de matrices de rigidité dynamique exacte. Ces éléments prennent en compte, s'il $\mathrm{y}$ a lieu, le couplage fluide-structure (coude, piquage, changement de section, ...). La résolution du problème nécessite un petit nombre d'éléments (un seul élément pour un tronçon droit quelle que soit sa longueur) et s'opère par assemblage et résolution du système linéaire respectant les équations d'équilibre aux jonctions entre les éléments. L'amortissement est introduit localement, ce qui permet de spécifier des valeurs différentes selon les éléments du circuit (supportage, tuyau,...).

\subsection{L'architecture du code CIRCUS}

Le code CIRCUS dispose d'une Interface HommeMachine adaptée aux études de tuyauteries par l'emploi du vocabulaire du métier. Les données sont définies selon le domaine d'étude (écoulement, mécanique, acoustique,...). Les configurations d'études sont gérées automatiquement par l'interface. Celle-ci permet de réaliser les calculs complémentaires (calcul de contraintes dynamiques, de durée de vie, ...) ainsi qu'une édition graphique des résultats sous forme de spectres ou de déformées. Cette approche permet d'effectuer l'ensemble des calculs d'une étude dans un environnement unique et avec un modèle unique. Cet aspect permet d'obtenir des diagnostics élaborés et de simuler alors l'impact de modifications, sans aucune perte d'information.

\section{- 3.3 La base de données acoustiques des matériels}

La formulation monodimensionnelle [6] utilisée permet de caractériser intrinsèquement un élément par deux entités décrites sous forme de spectre : une matrice représentant la propagation des variables d'état du problème, et un vecteur source correspondant à une discontinuité de ces variables.

Théoriquement, une seule matrice et un seul vecteur source suffisent à caractériser un élément. Cependant, dans la mesure où il exprime des phénomènes physiques distincts auxquels se rattachent des lois d'évolutions particulières, il est plus réaliste de traduire le comportement de l'élément par une succession de matrices et de vecteurs sources.

Une mise en forme générale des données a conduit à un formalisme unique permettant de décrire l'ensemble des caractéristiques intrinsèques des matériels. On définit des zones de validité des modélisations, ce qui conduit à fournir des bornes de variation à des paramètres indépendants et à des variables. Pour chaque zone de validité, les matrices et les sources sont décrites sous forme spectrale, soit par une formulation analytique faisant intervenir les paramètres géométriques et du fonctionnement, soit par des spectres issus d'essais sur site.

\section{IV $\square$ ÉTUDES VIBRATOIRES DE CIRCUITS DE TUYAUTERIES}

L'objectif des études menées sur les lignes et les piquages dans les conditions de fonctionnement concerne essentiellement la détermination et la comparaison des niveaux de contraintes obtenus dans le piquage et dans la ligne principale [8]. Le problème est alors de faire apparaître une valeur de contrainte dynamique sur le collecteur dégageant une marge suffisante vis-à-vis de la limite d'endurance du matériau. Si cette marge n'est pas suffisante une étude de modification du circuit est alors entreprise.

\subsection{Les risques associés au couplage fluide-structure à basses fréquences}

Les fluctuations de pression générées, essentiellement par les pompes lors des fonctionnements à faible débit, provoquent la mise en vibration des réseaux de tuyauterie par couplage dans les changements de direction et dans les changements de section. Or les réseaux de tuyauterie, même convenablement supportés, possèdent plusieurs modes de forte participation dans la bande de fréquence $1-50 \mathrm{~Hz}$, c'est-à-dire dans une zone où le niveau de fluctuations de pression est important. Les risques sont alors :

- les risques d'accrochage d'un mode acoustique avec un 
mode mécanique du collecteur ; dans ce cas les vibrations importantes du collecteur imposent une reprise d'efforts sur les ancrages,

- les risques d'accrochage d'un mode mécanique et/ou acoustique du collecteur avec un mode du piquage d'où un risque de fissuration du piquage par fatigue vibratoire,

- les risques d'accrochage d'un mode de piquage avec la fréquence de passage des aubes devant le bec de volute de la pompe. Ce risque peut être écarté par adaptation de la fréquence du piquage.

\section{- 4.2 Etudes à partir du diagnostic en exploitation}

Sur le circuit d'injection de Sécurité Basse Pression RISBP du palier nucléaire français 1300 MW [9], un mécanisme de fissuration d'un piquage était imputable à la mise en résonance du piquage avec un mode acoustique-mécanique du circuit de refoulement, situé vers $30 \mathrm{~Hz}$, lui-même excité par la source hydro-acoustique de la pompe fonctionnant à débit partiel sur la "ligne de débit nul". Ce mécanisme apparaissait sur certaines tranches d'un même palier et pas sur d'autres.

L'objectif de l'étude était d'expliquer les différences de comportement d'une tranche à l'autre et de qualifier une solution industrielle. La démarche a consisté à utiliser des résultats d'essais par intensimétrie acoustique (figure 3) pour déterminer des paramètres de calcul : les sauts de pression et de débit de la pompe, les conditions de réflexion acoustique aux extrémités du circuit et la vitesse du son (figure 4).

L'étude du circuit fait apparaître que le quatrième mode propre du collecteur est proche du premier mode propre du piquage et l'étude de sensibilité a mis en évidence une variation des fréquences propres du piquage due aux réalisations de montage (dispersion mécanique).

La qualification d'une solution technologique a été réalisée. La modification proposée consiste en une augmentation de l'épaisseur du collecteur et une diminution de la longueur d'allonge, ce qui a pour but de diminuer la contrainte sur le collecteur et d'augmenter la fréquence propre du piquage.

Enfin on a vérifié qu'il n'existait pas de risque d'accrochage entre le nouveau mode du piquage et un mode mécanique et/ou acoustique du collecteur. Cette vérification a été entreprise pour prendre en compte la dispersion mécanique de réalisation.

Lors de cette étude, on a vérifié que la formalisation des modèles de la base de données du code CIRCUS reproduisait à $10 \%$ près les sources acoustiques de la pompe IS-BP. A partir de l'identification sur site de la source acoustique de

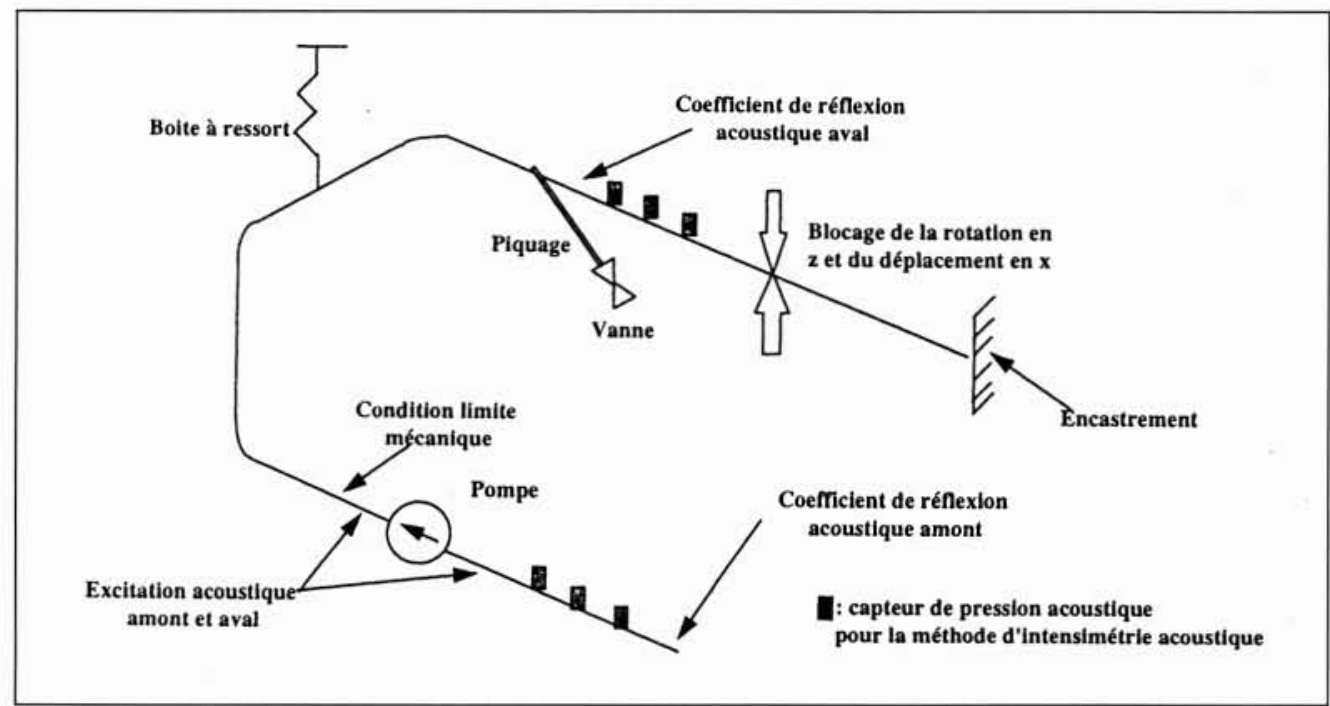

3. Instrumentation du circuit.

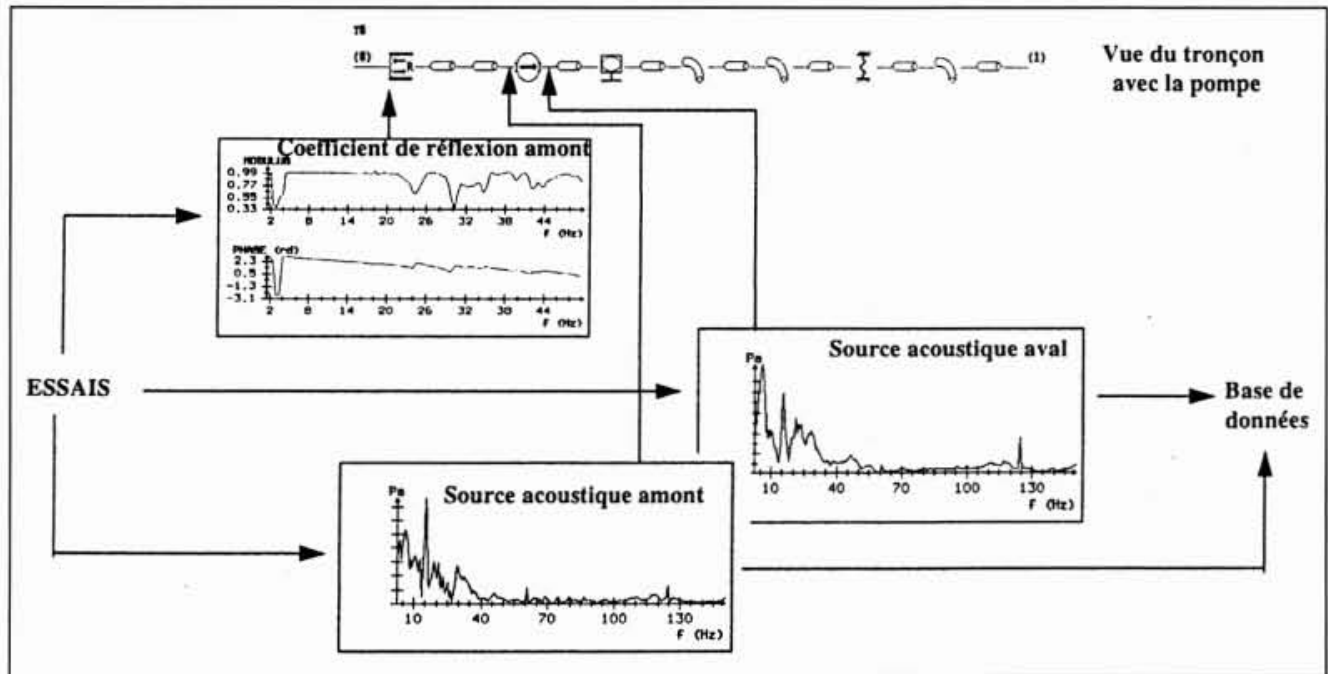

4. Modélisation CIRCUS des résultats d'essais. 


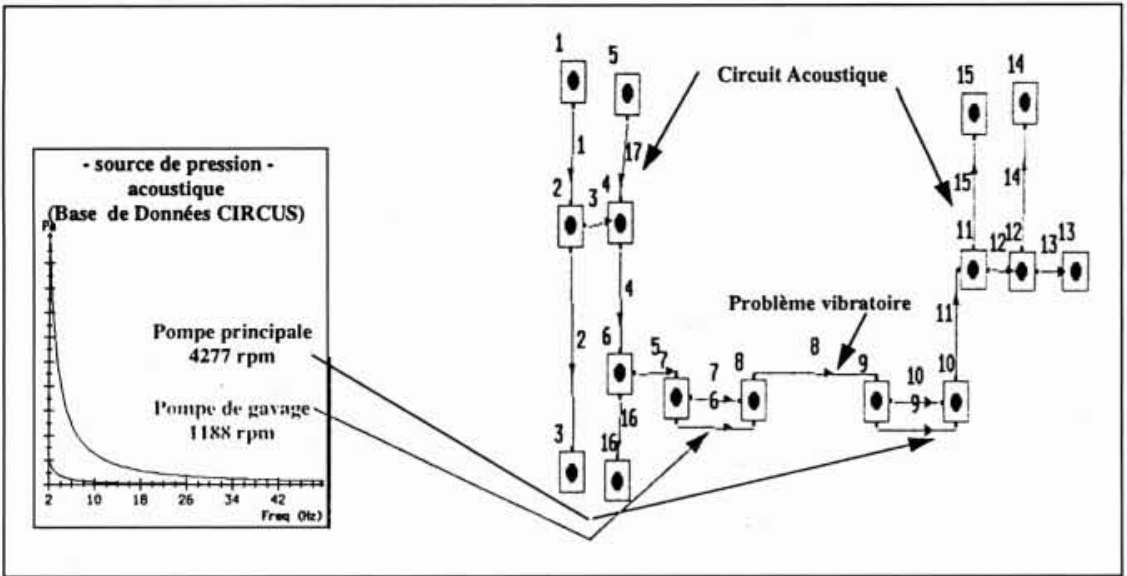

5. Sources d'excitation dues aux pompes et synoptique du circuit.

la pompe, l'amplitude calculée du déplacement vibratoire est comparable à celle mesurée et l'analyse a permis de retrouver les premiers modes vibratoires de la ligne de tuyauteries avec un écart inférieur à quelques pour-cent aux résultats de l'analyse modale réalisée sur site .

\section{- 4.3 Etudes à partir des données de conception du fonc- tionnement}

Les études $[10,11]$ du circuit d'injection de sécurité moyenne pression (RIS-MP) des paliers $1300 \mathrm{MW}$ et $1400 \mathrm{MW}$ font suite au constat de mouvement de collecteur (déplacements de valeurs élevées) sur la ligne reliant la pompe de gavage à la pompe principale du circuit. Ces vibrations dues aux sources de pompes fonctionnant à débit partiel sont des sources potentielles de dommages de fatigue vibratoire.

Le circuit inter-pompes est modélisé mécaniquement. Les deux parties acoustiques modélisées sont les circuits en amont de la pompe de gavage et en aval de la pompe principale du circuit. Le modèle mis en œuvre prend en compte les sources acoustiques des pompes et les effets d'interaction fluide-structure. Les sources d'excitation dues aux pompes n'ayant pas été caractérisées expérimentalement, c'est le modèle prévisionnel du comportement de la pompe de la base de données du code CIRCUS qui a été utilisé (figure 5). Le modèle est basé sur la théorie des matrices de transfert et prend en compte les paramètres géométriques et hydrauliques de la pompe. Les lois d'évolution des sources à l'aspiration et au refoulement sont constituées par les contributions d'une source correspondant au bruit de turbulence à basse fréquence et d'une source à la fréquence de passage des aubes.

D'un point de vue purement mécanique, seule la partie inter-pompes est à modéliser. Les conditions aux limites mécaniques sont les brides de raccordement des pompes que l'on peut considérer comme étant deux points fixes. Le collecteur est ainsi mécaniquement découplé du reste du circuit. Cette approche n'est plus valable dès lors que l'on prend en considération les effets de couplage fluide-structure car les conditions aux limites acoustiques au niveau des brides sont inconnues. Comme les ondes acoustiques se propagent audelà des pompes dans tous les éléments de tuyauteries en connexion directe avec la ligne, il est nécessaire d'inclure dans la modélisation tous les circuits en amont et en aval des pompes jusqu'aux organes d'isolement (vannes fermées, fonds, bâches,...) lesquels sont modélisés par des conditions aux limites acoustiques de pression acoustique nulle (grand bidon) et de débit nul (obturateur). De là, deux approches sont possibles. La première consiste à mener les calculs sur l'ensemble du modèle. Une autre solution consiste à partitionner l'ensemble des sous-systèmes (partie amont, interpompes, aval) de façon à élaborer par un calcul les informations correspondant aux conditions limites acoustiques aux extrémités du circuit inter-pompes. Cette seconde solution a été mise en œuvre dans [11], les calculs préliminaires sont réalisés sur les parties amont et aval afin d'estimer les coefficients de réflexion acoustique aux brides de pompes.

Pour l'étude menée sur le palier nucléaire français $1300 \mathrm{MW}$, la modélisation acoustique des tronçons amont et
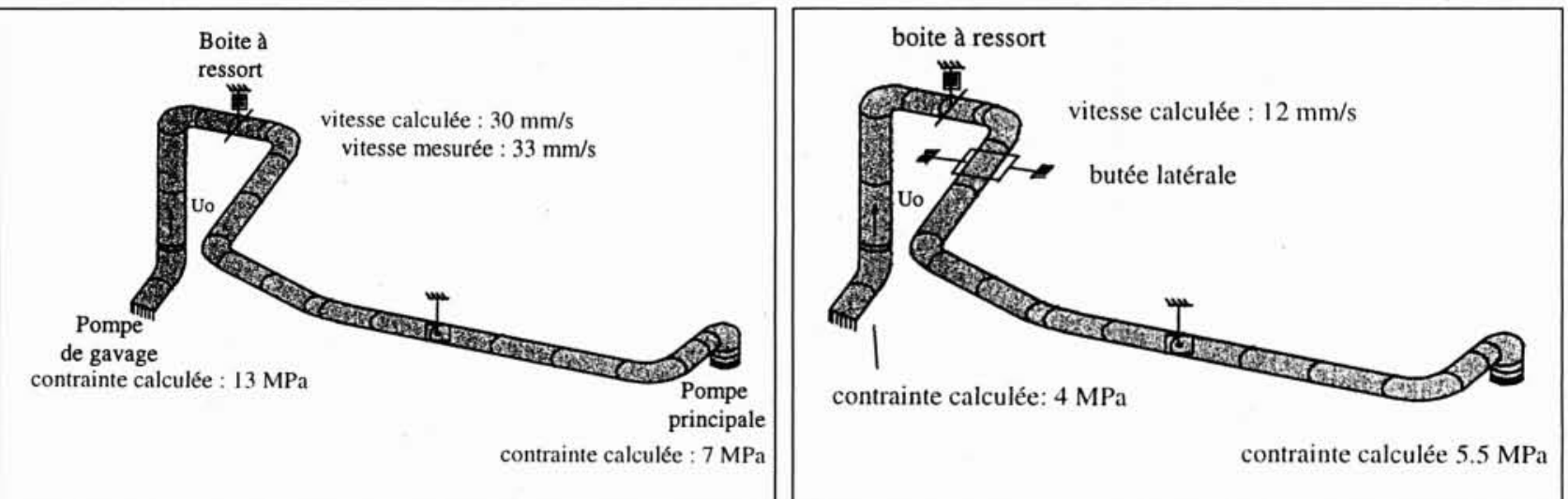

6a et $6 \mathrm{~b}$. Contrainte de Tresca maximale aux brides de pompes avant et après modification. 
aval a été construite avec les conditions limites acoustiques conformes aux conditions de fonctionnement lors de l'essai sur site. En l'absence d'indication d'essai sur site pour l'étude sur le palier nucléaire de $1400 \mathrm{MW}$, les tronçons acoustiques aval et amont ont été modélisés jusqu'à la bâche.

A partir de l'analyse modale expérimentale de la tuyauterie au repos effectuée sur le palier $1300 \mathrm{MW}$, les principaux paramètres du comportement mécanique ont été analysés afin d'élaborer un modèle du circuit inter-pompes mécaniquement recalé sur les principaux modes disponibles. Ce modèle sert alors de base au modèle couplé vibro-acoustique.

Les amplitudes calculées avec ce modèle sont comparables aux amplitudes mesurées lors de l'analyse en réponse de la tuyauterie en fonctionnement.

Afin de diminuer les niveaux vibratoires, il a été proposé de modifier le supportage du circuit inter-pompes RIS-MP du palier nucléaire français 1300 MW par l'adjonction d'une butée dans les directions latérale $\mathrm{X}$ et $\mathrm{Y}$. La modification intervient à l'endroit où la vitesse vibratoire de la ligne est maximale. La simulation de la modification met en évidence la forte diminution des vitesses vibratoires ainsi qu'une diminution d'un facteur 3 de la contrainte dynamique maximale au niveau de la bride de raccordement de la pompe de gavage (figures 6.a et 6.b). On observe également une amélioration de la répartition des niveaux vibratoires le long du tronçon inter-pompes. La zone la plus sollicitée du tronçon modifié est observée maintenant au niveau de la bride de raccordement de la pompe principale, le niveau de contrainte restant admissible. Par ailleurs, il a été vérifié que les fréquences du tronçon sont modifiées sans introduction de nouveau phénomène de couplage.

L'analyse en fatigue vibratoire par comparaison de la contrainte calculée à la limite admissible en fatigue a été réalisée. Les courbes de fatigue fournissant une valeur admissible en statique, il convient de prendre en compte le caractère dynamique du chargement et le caractère aléatoire des vibrations sous écoulement à travers un facteur de pic défini comme le rapport de la valeur efficace maximale sur la valeur efficace moyenne. Compte tenu du matériau de la tuyauterie, du facteur de pic et du facteur de concentration de contrainte, le seuil admissible est abaissé de $114 \mathrm{MPa}$ à $12 \mathrm{MPa}$

Pour le palier 1400 MW où une modification analogue a été étudiée, les mêmes conclusions ont été qualitativement obtenues. Les observations faites sur le modèle numérique donnent un ordre de grandeur cohérent avec les essais en fonctionnement effectués sur site. On constate une diminution très significative des vitesses vibratoires en amont de la butée rajoutée et une relative stabilité des niveaux calculés en aval de celui-ci. Néanmoins, on note une minoration par le calcul de certaines composantes de la vitesse vibratoire au voisinage de la butée. Une analyse modale du circuit sera réalisée et sera utilisée pour déterminer les conditions aux limites mécaniques des supports du tronçon ainsi que les niveaux de contraintes correspondants. Rappelons que même de très faibles mouvements réels de déplacement ou de rotation à l'endroit d'un point fixe peuvent influencer de manière importante les niveaux vibratoires à proximité.

\section{$\mathrm{V} \square \mathrm{CONCLUSIONS}$}

Le comportement vibro-acoustique des réseaux de tuyauteries en fonctionnement est un phénomène fortement couplé ; les mécanismes d'échange d'énergie entre l'écoulement, l'acoustique et la mécanique y sont complexes. Les différentes approches utilisées pour les études de vérification de la tenue de certains circuits nous ont amenés à développer des modèles pour les singularités acoustiques et mécaniques afin de mieux évaluer les critères et de compléter les règles utilisées lors de la conception des réseaux de tuyauteries. Le retour d'expérience de ces actions de recherche associées aux études est capitalisé au sein du code CIRCUS.

Par ailleurs, les études présentées montrent clairement l'importance des codes à base de connaissances des matériels et des chargements, particulièrement en dynamique.

En définitive, le code CIRCUS répond bien à un double besoin d'étude :

- en calcul prévisionnel, localiser et quantifier les phénomènes acoustiques ou vibratoires susceptibles de se propager. Il est alors possible d'évaluer les risques d'endommagement ou de faire évoluer le comportement du circuit en jouant sur ses paramètres de dimensionnement ou de fonctionnement,

- en calcul inverse, identifier les sollicitations dynamiques et les liaisons avec l'extérieur à partir de mesures effectuées sur une portion de circuit en service sur site. Des mesures intrusives permettent l'identification des sources hydro-acoustiques et des mesures non-intrusives (accéléromètres, jauges de contrainte), l'identification des sources acoustiques et mécaniques, ces dernières étant en cours de développement.

La base de données associée au code assure le retour d'expérience des modèles numériques et des données acquises sur site concernant les caractéristiques acoustiques et mécaniques des matériels.

\section{RÉFÉRENCES}

[1] Norme ANSI/ASME OM 3, (1982) “ Requierements for preoperational and initial start-up vibration testing of nuclear power plant piping systems".

[2] E. VATIN, J. GUILLOU, F. TEPHANY, C. TROLLAT *" Analyse numérique et expérimentale du comportement vibratoire d'un circuit de centrale nucléaire sous excitation hydraulique de sa pompe de circulation " Revue Générale Nucléaire " Année 1993 n 6 Novembre - Décembre.

[3] C. TROLLAT, F, TEPHANY, F, PAYAN, C. HILL - " Flow induced vibrations of piping systems : prediction of fatigue cracking for instrumentation nozzle of nuclear safety systems " American Nuclear Society - San Francisco 14-19 novembre 1993.

[4] JL TROLLE, E. VATIN “ Endommagement vibratoire des réseaux de tuyauteries " Revue EDF - EPURE n 44 - Octobre 1994.

[5] RCC-M : " Règles de Conception et de Construction des Matériels Mécaniques des îlots REP ". Edition juin 1988. Association française pour les règles de conception et de construction des matériels de chaudières électronucléaires.

[6] V. VILLOUVIER * Une base de données des singularités acoustiques en conduite. " 15ème Congrès International d'Acoustique - Trondheim, Norvège - juin 1995.

[7] JF LAURO, C. TROLLAT “ Modélisation hydro-acoustique des pompes centrifuges - lère Conférence international. " Bruit et vibrations des pompes "- Clamart, France - Juillet 1993.

[8] D. SELIGMANN, H. BOYELLE, F. PAYAN, F. TEPHANY “ Etude des vibrations de tuyauteries et des piquages : démarches, méthodes et modèles. "Séminaire de mécanique EDF à Aix-les-Bains, France, Octobre 1996.

[9] J. GUILLOU - " Étude du comportement vibratoire du circuit RIS-BP P'4 " - Note EDF/DER HP63/93/203/B

[10] D. SELIGMANN, J. GUILLOU - " Flow induced vibrations in a PWR piping system " SMIRT 13 - Porto Alegre 13-18 Août 1995

[11] F. PAYAN, Ch. BARATTE, D. SELIGMANN, J. GUILLOU “ Vibrations in piping systems " International Conférence on Vibration Problems (ICOVP)- North Bengal, Inde - Novembre 1996. 